\title{
Atmospheric Observation under Sampling Problem: The Impact of Unresolved Micro-Scale Boundary Layer Eddies on Climate Trends
}

\author{
Atoossa Bakhshaii, Edward A. Johnson \\ Biogeoscience Institute, University of Calgary, Calgary, Canada \\ Email: atoossa.bakhshaii@ucalgary.ca
}

How to cite this paper: Bakhshaii, A. and Johnson, E.A. (2017) Atmospheric Observation under Sampling Problem: The Impact of Unresolved Micro-Scale Boundary Layer Eddies on Climate Trends. Open Journal of Statistics, 7, 964-971. https://doi.org/10.4236/ojs.2017.76068

Received: November 7, 2017

Accepted: December 5, 2017

Published: December 8, 2017

Copyright $\odot 2017$ by authors and Scientific Research Publishing Inc. This work is licensed under the Creative Commons Attribution-NonCommercial International License (CC BY-NC 4.0).

http://creativecommons.org/licenses/by-nc/4.0/

\begin{abstract}
The observed basic weather variables are the main representative of climate trends and the atmosphere. The unresolved meteorological scale in weather observation such as micro scale, can produce a noticeable bias in amplitude, frequency, phase and climate trend of each observed variable time series. The bias in climate trend due to a small scale eddy can be as high as the amplitude of the eddy which could be greater than $1^{\circ} \mathrm{C}$ in a temperature trend. Such biased measurements of the state of the atmosphere limit all climate related studies.
\end{abstract}

\section{Keywords}

Weather Observation, Signal Analysis, Fast Fourier Transform, Eddy

\section{Introduction}

The earth and its surrounding atmosphere change constantly. The changes in our atmosphere are mainly (considering urbanization, land use change and atmospheric chemistry as noise relative to the rest of solar system harmonics) driven by forces initiated by the earth's position and movement in the solar system and the resulting incoming solar radiation. The incoming solar energy to the earth system varies with a few harmonics. These harmonics are not limited by but originated from the following facts [1]. 1) The earth rotates around its own axis every 23 hours, 56 minutes and 4 seconds. 2) The earth's rotation around the sun is about 365 days, 6 hours, 9 minutes and 10 seconds long (one sidereal year) or 365 days, 5 hours, 48 minutes and 46 seconds long (tropical year), but it does not return to its first position. 3) The plane of the Moon's orbit about the earth rotates with a period of 18.6 years and causes the earth to nod. 4) The pe- 
riod of rise and fall in sunspot activities is about eleven years on average while the sun itself has a longer noticeable cycle of 200 years. 5) The earth's orbit drifts up and down relative to its present orbit with a cycle of around 70,000 years. 6) The earth's orbit also moves relative to the orbits of the other planets with a period of about 100,000 years. 7) The major axis (eccentricity) of the earth's orbit changes almost every 100,000 years while the semi-major axis of the orbital ellipse remains unchanged; this could result in a $6.8 \%$ change in incoming solar radiation. 8) The earth's axis traces a circle in the sky over 26,000 years. 9) The tilt of the earth's axis varies from 22.1 degrees to 24.5 degrees every 41,000 years. Although we have no doubt that the solar energy, incoming radiation and earth movement play a main role in the climate change signal, our observations do not clearly reflect the connection. Could the observation be wrong? In the next paragraph we explain how atmospheric science has been focused on the scale of horizontal motion in space more than a frequency base. Although it is not proper to regard a scale of motions as independent of the smaller-scale or larger-scale in physics and atmospheric science, it has been done for practical reasons such as the limited available observations.

The simple yet remarkable famous question "Does wind have a speed?" was raised by Lewis Fry Richardson due to the difficulty that he and other scientists of the time had in measuring the horizontal divergence with sufficient accuracy. Richardson performed a numerical integration of the governing equations of motion, which had been suggested by Bjerknes earlier, over a horizontal grid of about $200 \mathrm{~km}$ and four vertical layers of approximately $200 \mathrm{hPa}$, centered over Germany. He used the observation made at 7 UTC (Universal Coordinate Time) on May $20^{\text {th }}, 1910$. He computed the time derivative of the pressure in central Germany between 4 and 10 UTC. Although the predicted 6-h pressure changes had huge discouraging errors [2]. Richardson's idea laid the foundation of $\mathrm{Nu}$ merical Weather Prediction (NWP) and modern data assimilation today.

It becomes obvious today that the inadequacies of observation alone would have condemned any attempt, of which Richardson was well aware. Besides, we know today, the fastest traveling signal (sound waves) in his experiment travels at about $300 \mathrm{~m} / \mathrm{s}$ and the integration needed to be done faster in a smaller time increment (about 10 minutes). The experiment failed due mostly to the fact that the initial conditions were not balanced [2]. Charney [3] and Eliasen [4], instead of attempting to deal with the atmosphere in all its complexity, used a simplified model approximating dominant large motion of the atmosphere by filtering the fast sound waves. The division of scale of motions came to attention in an analytical way. They had successfully predicted the pressure field with the cost of eliminating vertical motion and acceleration. In other words they filtered the small scale waves to predict the larger scale of motion based on practical model deficiency and error in the observation. Nowadays the state of the art observations and numerical forecasting have advanced enough to predict the atmosphere about two weeks in advance in a predictable condition [2]. 
Atmospheric scientists know that when a front is involved the scale is about hours to days in time and $100 \mathrm{~km}$ to $1000 \mathrm{~km}$ in space (meso- $\alpha$ scale). When the heat exchange via turbulence is relevant the scale of space changes to $1 \mathrm{~mm}$ to 1 meter and time to seconds (micro- $\sigma$ scale). Figure 1 summarizes a schematic of atmosphere scales based on horizontal motions and time. The American Meteorological Society and World Meteorological Organization have slightly different division of scales of motion. Although one must know there is no definite border line between the motions and it is not proper to regard a scale of motions as independent of the other atmospheric scales of motions, it has been done for practical reasons such as the limited available observations. The scale of motions and the observation limitation often are overlooked when the target of study involves climate change.

The disconnection between the data and natural solar system frequencies can be partially explained by the short length of available data and biases in our measurements. Weather observation and weather practice evolved based on instrument availability and resources more than on classical sampling theory. The objective of this paper is to explain the climate uncertainty that is caused by high frequency turbulence in the atmosphere. How do the biases cause some predictions to fail and some not. How do the sampling frequencies and down sampling (such as monthly mean) add bias in our observations and compromise some climate trends.

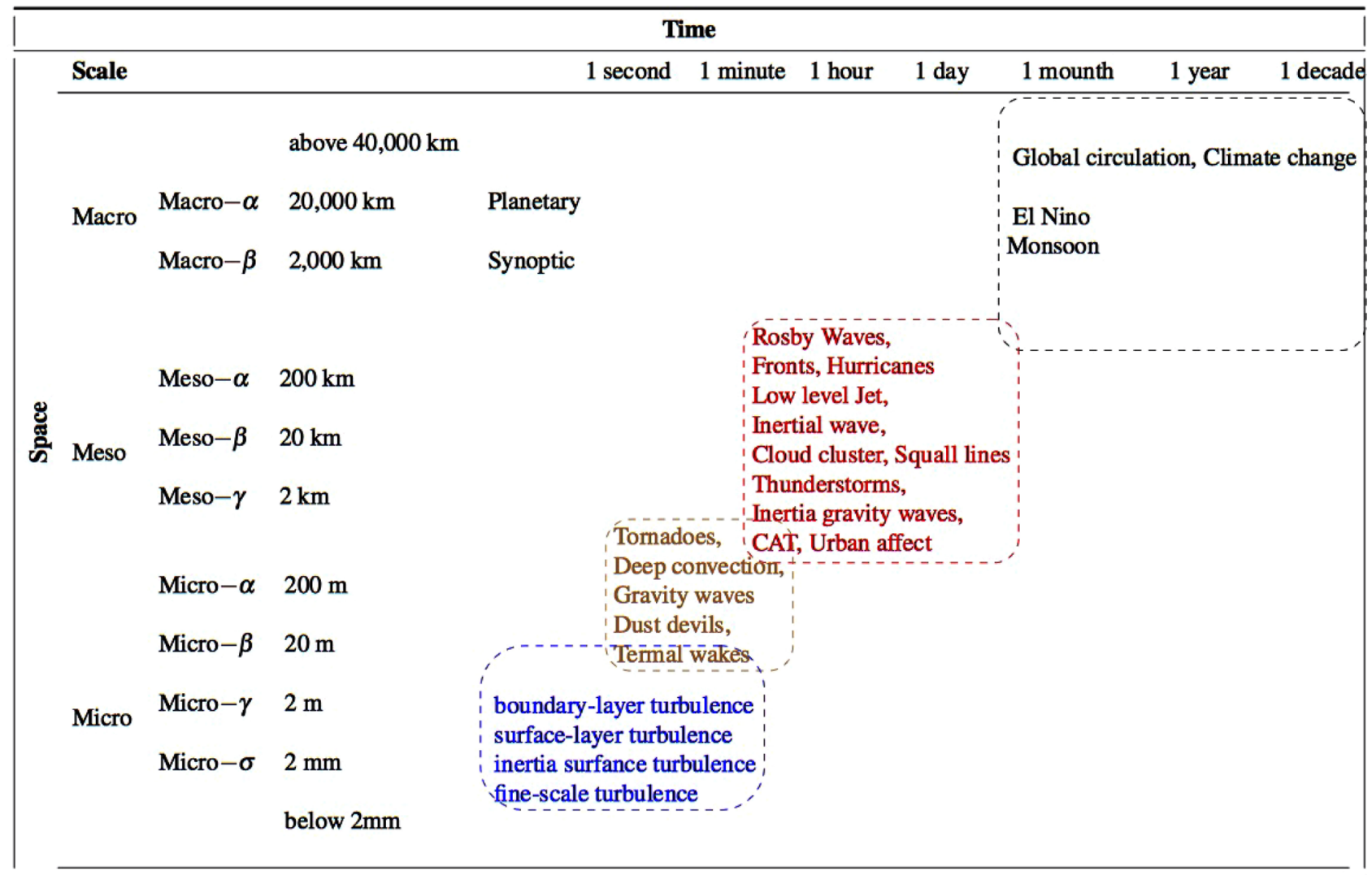

Figure 1. Scale of horizontal motion in the Atmosphere. 
The Nyquist-Shannon [5] sampling theorem is widely used in many fields as a fundamental bridge between continuous-time signals (analog signals) and discrete-time signals (digital signals). It simply says: if there is a signal that is perfectly band limited (limited to a certain high frequency) to a bandwidth of $f_{0}$, then it can be collected with all the information in the signal by sampling at discrete times, as long as the sample rate is greater than $2 f_{0}$. Unfortunately, while the theorem is simple and straightforward, it can be very misleading when one tries to apply it to the atmosphere. One of the challenges is the fact that in nature we rarely have a perfect band-limited signal and aliasing (an artifact caused when different signals become indistinguishable) occurs. To avoid aliasing, we need to sample the signal faster than the highest frequencies or at least filter (low pass filter) the signal prior to sampling to remove the highest frequencies as much as we can, although filtering might change the phase and amplitude of a signal.

In designing sample-time or sample-space systems, the variables that we need to consider are signal accuracy (phase, amplitude and frequencies are equally important in atmospheric measurements) and various kinds of system cost (power consumption, dollar cost, etc.). Increasing the signal sample rate will always increase the signal accuracy. To sample the atmosphere based on the Nyquist-Shannon sampling theorem we need to record weather observations faster than every second in time and at least every $50 \mathrm{~m}$ in space, if we assume the band limits of atmospheric signals are about micro- $\alpha$ (Figure 1). The current fastest available boundary layer weather observation is hourly observation of basic weather parameters which are not evenly distributed in space. Hourly observation filters the higher frequency by averaging samples between hours. The filter is an automated algorithm that is applied via data loggers at the weather stations and the average hourly data is archived at a national center. In the following section we use an artificial sinusoid signal to demonstrate the impact on a climate trend of the bias of a high frequency signal that was filtered at the sampling time. We found the bias of unresolved scale of motion can change the magnitude and the slope of climate trend.

\section{A Practical Test to Demonstrate the Impact of the Bias of Micro Scale Observations on Macro Scale}

As there is no access to long time high frequency observations and to the real state of the atmosphere, we used a simple sinusoid wave as representative of a harmonic atmosphere. We considered that air temperature $(2 \mathrm{~m}$ air temperature for example) at one station is a composition of 7 sinusoid harmonic signals with equal phase and wave numbers as presented by Equations (1) to (3). The wave number $(K)$ is equal to 1 and the phase $(\varphi)$ is zero for all harmonics. These seven harmonics are representative of turbulence, hourly, daily, monthly and annual temperature changes. A day is considered to be 23.9 hours and a month 29.5 days while a year is 365.25 days (as explained in the introduction). 


$$
\begin{gathered}
\operatorname{Signal}(t)=\sum_{i=1}^{7}(\operatorname{amplitude}(i) \times \sin (2 \pi K \text { frequencies }(i) \times t+\varphi(i))) \\
\text { amplitude }=[3,1,1.5,4,6,18]
\end{gathered}
$$

Periods

$=$ [2.5 seconds, 1.0001 minutes, 30 minutes, 0.5 day, 1 day, 1 month, 1 year $]$

Then the simplified temperature signal is sampled every 5 seconds and averaged every 720 samples to present the hourly temperature in the same fashion as Canadian data loggers sample the air temperature at automated stations. The five second sampling is common among Canadian automated stations and many other weather stations. The C-1 climate station in Niwot Ridge, Colorado, USA records the peak wind gust information instantaneously with a sampling interval of 5 seconds, and the other measurements are one hour averages comprised of 720 samples collected at 5 second intervals. At North Dakota Agriculture Weather Network (NDAWN) wind speed and direction are measured every 5 seconds and are averaged hourly and daily. Air temperature, relative humidity, solar radiation, bare soil temperature, and turf soil temperature are measured every 60 seconds and are averaged hourly. The hourly artificial temperature from the sampling is compared with the original signal (Figure 2). The hourly artificial temperature is different from the original signal and the difference in this very simplified case was mainly due to the first harmonic in artificial temperature signal which is representative of unresolved turbulence in our measurements. Figure 2(a) and Figure 2(b) show two signal time series and the growing bias in the sampled signal. The annual and long term trends also differ in this particular example. The difference is comparable with the amplitude of the first harmonic and it changed at the rate of 0.002 per year. The bias changes by the characteristics of the unresolved first harmonic such as frequency and amplitude. Table 1 shows a variation of biases and trends when the first harmonic's frequencies changed in the example. The largest difference in trend was observed when the normal frequency is an integer. That can be explained by Figure 3 which shows the first harmonics by different sampling speed. The first harmonic considered a turbulence with a frequency of $0.4 \mathrm{~Hz}$ and amplitude of 3 $\left(3^{\circ} \mathrm{C}\right.$ ). The slowest sampling rate is $0.2 \mathrm{~Hz}$ (Figure $3(\mathrm{c})$ ) and the normal frequency is 2 , which falls to an integer normal frequency class (Table 1). The slowest sampling rate creates an alias that the amplitude will increase by the order of $10^{-11}$ per hour (Figure $3(\mathrm{c})$ ).

We also tried to calculate the daily and monthly average based on a calendar when days are 24 hours and a year is 365 days. Then we compared the trend of monthly average with the 5 second sampling and 1 second sampling trends. Figure 2(c) shows that the two hourly averaged trends are apart and the monthly trend has a completely different slope. What the figure does not clearly present is the true signal and the slow sampled signal trends are not parallel so the error grows by time (Table 1 ).

The above example is a simplified case with just 7 harmonics and no noise 


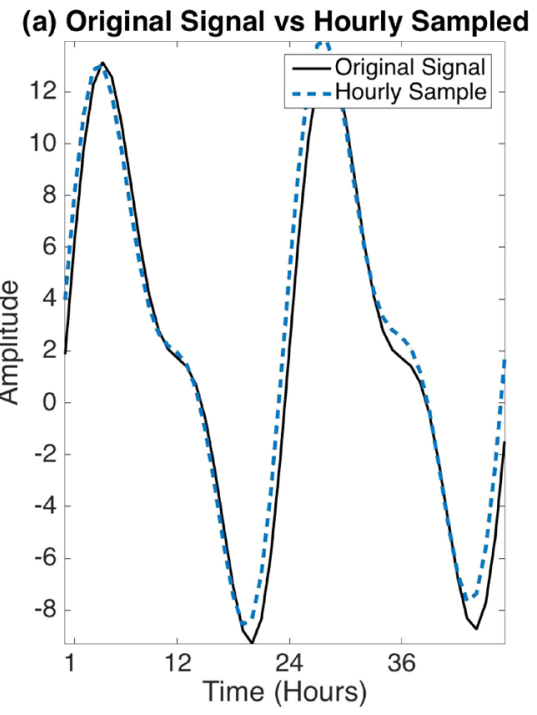

(b) Bias

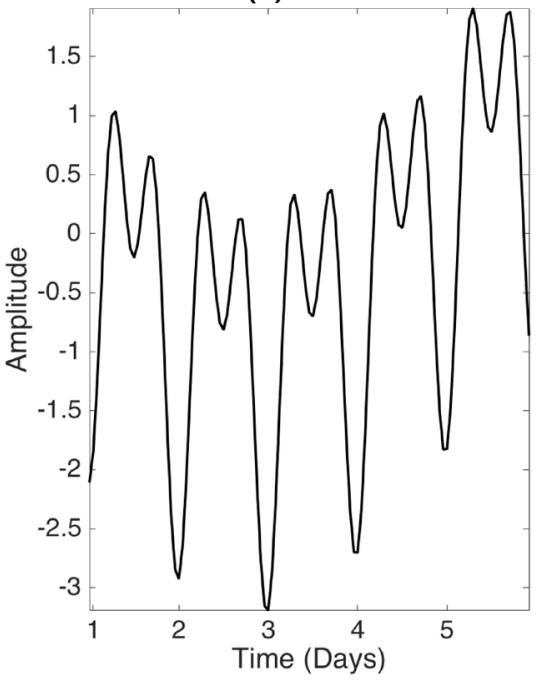

(c) Trends

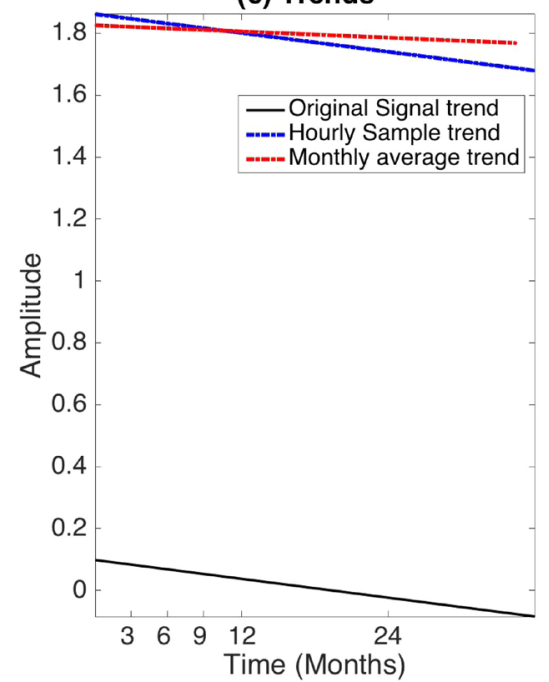

Figure 2. The artificial temperature time series that is a composition of six harmonics expressed by Equations (1) to (3).

(a) First harmonic (Sampling frequency $20 \mathrm{~Hz}$ ) (b) First harmonic (Sampling frequency $1 \mathrm{~Hz}$ ) (c) First harmonic (Sampling frequency $0.2 \mathrm{~Hz}$ )
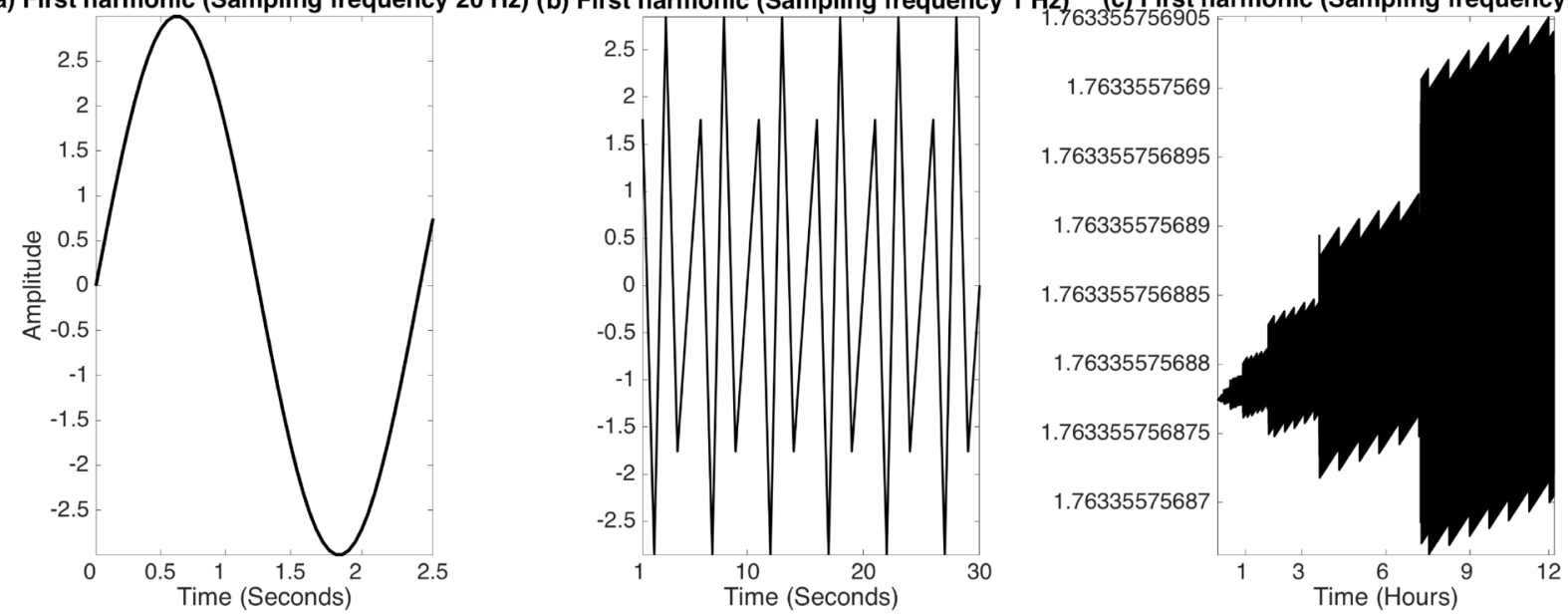

Figure 3. The first harmonic of the artificial temperature sampled by three different speeds.

Table 1. The variation of bias by the first harmonic's frequency. The normal frequency is the ratio of $f_{\text {first harmonic }} / f_{\text {sampling }}$ and the bandwidth is the highest frequency in the signal composition.

\begin{tabular}{cccc}
\hline Normal frequency $\left(\mathrm{f}_{0} / \mathrm{f}_{\mathrm{s}}\right)$ & Bandwidth $\left(\mathrm{f}_{0}\right)$ & Average annual bias & Annual trend difference \\
\hline 0.04 & 0.008 & -0.0008 & -0.0015 to -0.0089 \\
0.3 & 0.06 & 1.1038 & -0.0015 to -0.0089 \\
0.4 & 0.08 & 1.4470 & -0.0015 to -0.0089 \\
0.5 & 0.1 & 1.7628 & -0.0015 to -0.0089 \\
0.6 & 0.12 & 2.05 & -0.0015 to -0.0089 \\
1 & 0.2 & -0.0006 & -2.8546 to -2.8443 \\
2 & 0.4 & -0.0006 & -1.7648 to -1.7544 \\
3 & 0.6 & -0.0005 & +1.7619 to +1.7723 \\
5 & 0.8 & -0.0005 & +2.8521 to +2.8621 \\
& 1 & -0.0005 & -0.0015 to -0.0089 \\
\hline
\end{tabular}


while the real atmosphere is a continuous spectrum of harmonics and variation of noises such as industrial pollution and urbanization. The distribution of atmospheric measurements in space and time is far behind any sampling theory and meanwhile archiving and down sampling are based on calendar frequencies rather than natural frequencies.

\section{Conclusions}

Atmospheric measurements and atmospheric science constantly fall behind due to the limitation and quality of data. There is no doubt that increasing the number of observations in horizontal space and time is the ultimate solution. Meanwhile the sampling can be improved by considering: 1) magnitude and frequencies of local unresolved scale of motions (higher frequencies than sampling rate turbulence); and 2) down sampling based on real existing frequencies rather than the conventionally agreed frequencies. Considering the direct relation of scales in space and time of a phenomenon of interest and limitation of our available data to resolve the long term trend of such a phenomenon is crucial. In cases where the climate data cannot resolve the spatial = temporal scale, we use NWP models and data assimilation or available analysis. The combination of knowledge of mechanism and data through high resolution analyses can be a temporary alternative.

The observed weather variables are used in many fields including biology, civil engineering, hydrology, ecology, finance and meteorology. Although we learned from Lorenz [6] that any small uncertainty in the current state of the atmosphere can lead to chaos in our predictions, we haven't removed the uncertainty in our observations completely. In the absence of knowledge of the atmospheric state, the vast human efforts to overcome the problem are constantly underestimated and the estimated time series trends are overestimated by users.

\section{Acknowledgements}

The research was supported by a Natural Science and Engineering Research Council of Canada Discovery Grant to Edward A. Johnson. Many thanks to Prof. Kiyoko Miyanishi for her comments.

\section{References}

[1] Stull, R. (2016) Practical Meteorology: An Algebra-Based Survey of Atmospheric Science. University of British Columbia, British Columbia, 797-801.

[2] Kalnay, E. (2003) Atmospheric Modeling, Data Assimilation and Predictability. Cambridge University Press, Cambridge.

[3] Charney, J.G. (1951) Dynamic Forecasting by Numerical Process. American Meteorological Society, Boston, 470-482. https://doi.org/10.1007/978-1-940033-70-9_40

[4] Eliasen, E. and Machenhauer, B. (1965) A Study of the Uctuations of the Atmospheric Planetary ow Patterns Represented by Spherical Harmonics. Tellus, 17.

[5] Shannon, C.E. (1998) Communication in the Presence of Noise. Proceedings of the 
IEEE, 86, 447-457. https://doi.org/10.1109/JPROC.1998.659497

[6] Lorenz, E.N. (1963) Deterministic Non-Periodic ow. Journal of the Atmospheric Sciences, 20, 130-141.

https://doi.org/10.1175/1520-0469(1963)020<0130:DNF>2.0.CO;2 